\title{
A 70-year-old male with peripheral neuropathy, ataxia and antigliadin antibodies shows improvement in neuropathy, but not ataxia, after intravenous immunoglobulin and gluten-free diet
}

\author{
Dharshan \\ Anandacoomaraswamy' \\ Jagdeesh Ullal² \\ Aaron IVinik ${ }^{2}$ \\ 'Department of Internal Medicine, \\ Coney Island Hospital, Brooklyn, \\ NY, USA; ${ }^{2}$ Strelitz Diabetes Center, \\ Department of Internal Medicine, \\ Eastern Virginia Medical School, \\ Norfolk, VA, USA
}

\begin{abstract}
This is a case of a 70-year-old man with severe peripheral neuropathy, type 2 diabetes and progressively worsening cerebellar ataxia. He was found to have circulating antigliadin and antireticulin antibodies compatible with celiac disease in the absence of intestinal pathology. The peripheral neuropathy improved with a gluten-free diet, antioxidants and intravenous immunoglobulin, whereas the ataxia did not. This case illustrates the need to test for celiac disease in patients with idiopathic ataxia and peripheral neuropathy and the need for alternative therapies for ataxia.
\end{abstract}

Keywords: celiac disease, peripheral neuropathy, autoimmune disease, cerebellar ataxia, type 2 diabetes

\section{Introduction}

The combination of ataxia and peripheral neuropathy (PN) may be associated with a variety of conditions such as paraneoplastic syndromes, celiac disease (CD), Sjogren's syndrome, chronic inflammatory demyelinating polyneuropathy, and Miller-Fisher syndrome, (Honnorat et al 2001; Lang et al 2003; Koller et al 2005). CD is a multigenetic T-cell mediated autoimmune disease where intolerance develops to the dietary protein 'gluten'. CD typically presents with gastrointestinal manifestations but in some cases ataxia can be the presenting sign. Other extraintestinal manifestations include iron-deficiency anemia, osteoporosis, cryptogenic hypertransaminasemia, recurrent abortion, autoimmune diseases which are now recognized to be more common in the absence of gastrointestinal symptoms in patients with gluten-sensitive enteropathy. It has been estimated from prevalence studies that for every patient with CD who presents with gartrointestinal symptoms, there are eight patients with silent disease. Since diabetes is a major cause of PN (Dyck et al 1993; Vinik et al 2006) there is complacency about seeking alternative causes of neurological syndromes in such patients. Autoimmune pathogenetic mechanisms have been implicated in neuropathy in diabetes (Granberg et al 2005). It is unclear whether the antibodies are pathogenic or innocent bystanders (Vinik et al 2005a), but there is little doubt that there is a greater frequency of inflammatory demyelinating neuropathies in people with diabetes than in the general population (Sharma et al 2002). The association of cerebellar ataxia and PN in diabetes has been reported in a patient with circulating GAD antibodies (Iwasaki et al 2001). The prevalence of $\mathrm{CD}$ in children with juvenile diabetes is at least $4.6 \%$ (Aktay et al 2001) and is known it is known to be a hall mark of type- 1 diabetes. However, CD may occur in type 2 diabetes or in latent autoimmune diabetes of adult (Sanchez et al 2007). Thus, it is not surprising to find CD in older males with diabetes. We present a case of cerebellar ataxia associated with $\mathrm{CD}$ without gastrointestinal involvement in a 
patient with well-controlled diabetes in whom treatment of the autoimmunity reversed the PN, but not the ataxia.

\section{Case report}

A 70 -year-old Caucasian male with a $3 \frac{1}{2}$ year history of type 2 diabetes, presented with complaints of unsteadiness. He had numbness in his feet and erectile dysfunction. He took irbersartan for hypertension and had a strong family history of type 2 diabetes but no autoimmune diseases.

His musculoskeletal system showed bilateral mild wasting of the quadriceps and total loss of vibration sensation in the feet. Pinprick sensation was reduced in both his lower limbs to the level of the ankles but only a mild reduction in the upper limbs. Tendon reflexes were intact. Romberg's sign was negative. Tandem stand test was strongly positive as was the one-legged stand test. Two months later he exhibited gait ataxia, hypermetria, and intention tremor.

Extensive testing for possible causes was done. Paraneoplastic antibodies, urinary heavy metal excretion, serum electrophoresis, ANA, RPR, and HIV tests were negative. A MRI of the spine and brain did not reveal any pathology, PET scan of the lungs for occult malignancy was negative and CSF was normal. Vitamin E and vitamin $\mathrm{B}_{12}, \mathrm{TSH}$, ceruloplasmin, and lactic acid levels were all normal. His HbA1c was $6.4 \%$.Since this clinical presentation was distinctly unusual for diabetes, we investigated further for auto-immune neuropathy and incubated his serum with N1E-115 neuroblastoma cells which showed apoptotic cell death confirming the autoimmune etiology (Pittenger et al 1997).

Electromyography showed supranormal conduction velocities over the peroneal, tibial, medial, ulnar, and sural nerves but low amplitudes indicating a mild axonal form of neuropathy. Quantitative sensory testing (QST) revealed a mild large-fiber neuropathy with impaired vibration perception and relatively intact warm and cold thermal perception in the feet. All these findings were compatible with a metabolic disease such as diabetes. Hence, diet, exercise and oral hypoglycemic therapy were initiated to control the diabetes and in light of the strongly positive effects of his serum on immunotoxicity assay, we initiated intravenous immunoglobulin (IVIg) therapy along with oral antioxidant therapy (Vitamin C, $500 \mathrm{mg}$ /day; Vitamin E, $400 \mathrm{IU} /$ day; alphalipoic acid, $1200 \mathrm{mg} /$ day; and dihomogammalinolenic acid, $3900 \mathrm{mg} /$ day). The magnetic resonance scan of his brain revealed evidence of moderate cerebellar atrophy.

Six months later, his neuropathy had improved except for mild impairment of vibration perception. However, cerebellar signs were still present. By this time he had completed two infusions of intravenous immunoglobulin $($ IVIg) $(1 \mathrm{~g} / \mathrm{kg} /$ day $\times 2)$ but unfortunately he developed a centripetal rash after the 2nd infusion, and no further IVIg was given. The patient's serum remained toxic to neuronal cells in culture despite the administration of IVIg. Table 1 shows the improvement in QST and autonomic nerve function tests at presentation and 17 months into treatment.

These progressively worsening cerebellar signs were thought to be due to an autoimmune process, as the serum was very toxic to neuroblastoma cells. Antigliadin IgG, antigliadin IgA and antireticulin antibodies were found to be positive among many other antibody panels that were investigated. Other antibodies including, paraneoplastic antibodies, anti-GAD antibodies, antithymoglobin, antimicrosomal, anti-R1, anti-Ma/Ta, antiendomysial antibodies, anti-Yo-antibody, anti-Hu antibody, antineural antibodies (ANNA-1 and ANNA-2) and Lyme titers were negative (these tests were performed by LabCorp, Burlington, NC). Small bowel studies including esophagogastroduodenoscopy and biopsies of small bowel failed to show any evidence of CD. Though there was no biopsy evidence of CD, he was advised to embark on a gluten-free diet based on case reports that suggested that ataxia could be the sole manifestation of $\mathrm{CD}$ in the absence of gastrointestinal pathology and based on antibody positivity. There was also the possibility that the intestinal biopsy had missed the affected site.

After 2-months on a gluten-free diet, his peripheral neuropathy showed further improvement but he still had all the signs of cerebellar dysfunction supporting the notion of different mechanisms for the cerebellar ataxia and the peripheral neuropathy. After 20 months on the diet, all the celiac antibody titers became normal and the PN showed progressive improvement but the cerebellar function did not. Figure 1 shows the clinical improvement over time.

\section{Discussion}

This patient has progressive cerebellar ataxia, improving peripheral neuropathy and decreasing titers of celiac antibodies. Hence, it is compelling to consider CD as the cause of his condition. Cerebellar ataxia could be the only manifestation of an otherwise occult CD in this patient. Sensory axonal neuropathies and small fiber polyneuropathies are the most frequently reported types of peripheral neuropathy in CD (Chin et al 2003). There are reports to suggest that ataxia could be the sole manifestation of CD especially in people with a genetic susceptibility (Hadjivassiliou et al 1998; Bushara et al 2001). There is growing evidence of 
Table I Quantitative measures of peripheral sensory threshold indices and autonomic function done at the time of presentation and after 17 months of treatment. Measures of peripheral neuropathy are vibration and pressure sensations, warm and cold perceptions and painful perception (Vinik et al 1995). The E/I (exipiratory/ inspiratory ratio), 30:15 stand test and valsalva are measures of autonomic nerve function (Vinik and Erbas 200I)

\begin{tabular}{llll}
\hline $\begin{array}{l}\text { Neuropathy } \\
\text { t tests(units) }\end{array}$ & At presentation & After I7 months & $\begin{array}{l}\text { Normal values } \\
\text { for }>\mathbf{9 0 \%} \text { specificity }\end{array}$ \\
\hline Vibration (volts) & 9.11 & 3.63 & $0-6.1$ \\
Pressure (log g) & 4.13 & 3.58 & $0-3.95$ \\
Cold perception $\left({ }^{\circ} \mathrm{C}\right)$ & 8.6 & 9.2 & $0-10.5$ \\
Warm Perception $\left({ }^{\circ} \mathrm{C}\right)$ & 10.3 & 9.3 & $0-12.4$ \\
Cold pain $\left({ }^{\circ} \mathrm{C}\right)$ & 24 & 11.1 & $0-23.3$ \\
Warm pain $\left({ }^{\circ} \mathrm{C}\right)$ & 15.5 & 10.4 & $0-17.7$ \\
E/l & 1.11 & 1.06 & $>1.08$ \\
Valsalva & 1.57 & 1.21 & $>1.15$ \\
$30: 15$ ratio & 1.13 & 1.05 & $>1.08$ \\
\hline
\end{tabular}

the association of $\mathrm{CD}$ with many neurological disorders (Hadjivassiliou et al 1996). These conditions include cerebellar ataxia, PN, epilepsy, dementia, and depression. Among these, cerebellar ataxia, PN and memory impairment are the most commonly seen associations (Hadjivassiliou et al 1998; Luostarinen et al 1999; Pereira et al 2004). There is also some suggestion that migraine, encephalopathy, chorea, brain-stem dysfunction, myelopathy, mononeuritis multiplex and Guillian-Barre like syndrome could be the presenting extra-intestinal manifestations of $\mathrm{CD}$ with or without intestinal pathology (Bushara 2005).
Although ataxia is believed to be due to immunological damage to the cerebellum, posterior column, and peripheral nerves (Hadjivassiliou et al 1998), it is unclear whether there is a common pathogenic mechanism that affects both the nervous system and the gastrointestinal tract. Ataxia in CD can be due to malabsorption and associated vitamin deficiency (Hagen et al 2000). There is a report where parenteral vitamin E administration improved the clinical and neurophysiological signs of CD in a vitamin E-depleted patient with this disease (Battisti et al 1996). However, our patient had normal Vitamin $\mathrm{E}$ levels and had received vitamin E.

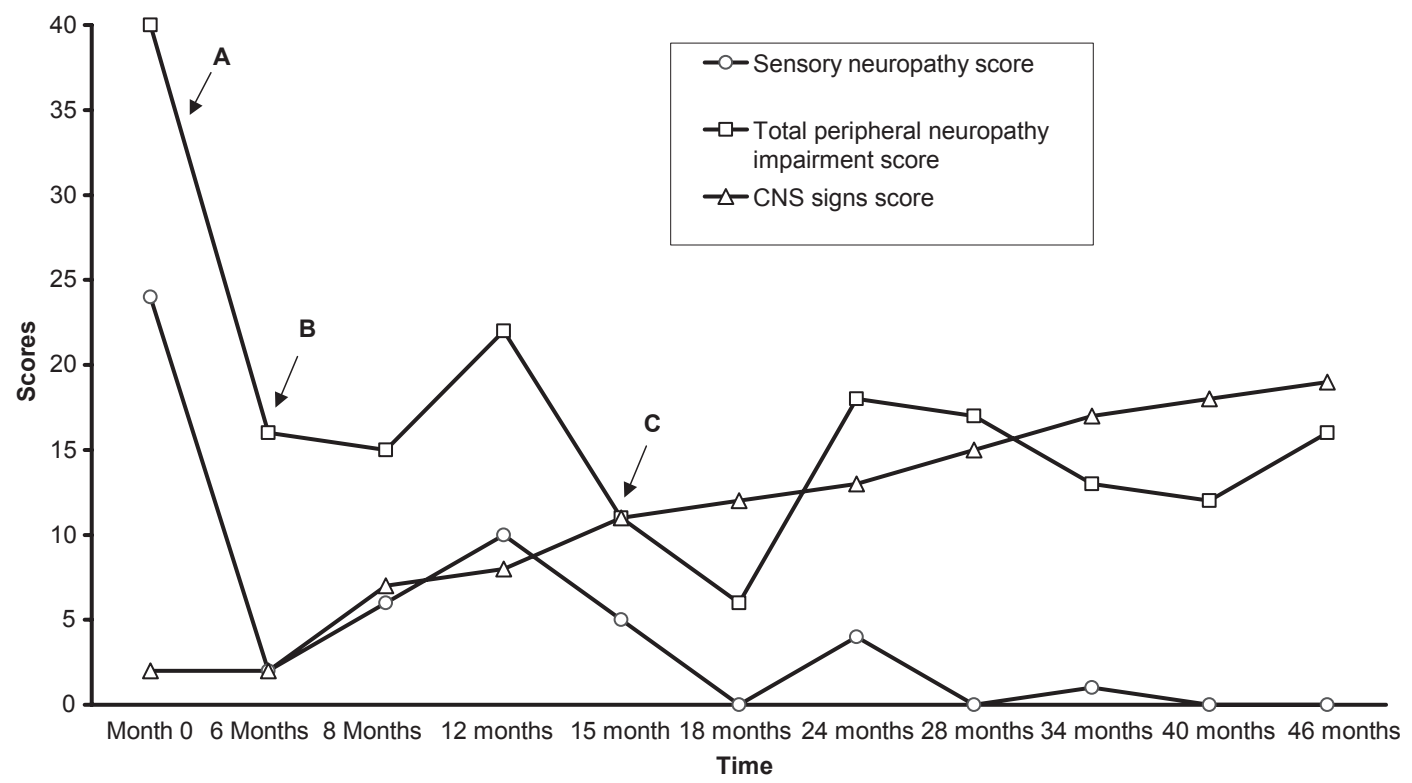

Figure I This figure represents the progressive changes in central and peripheral nervous system scores. The central nervous system (CNS) signs score is the sum of the number of positive cerebellar signs. The total peripheral neuropathy impairment scores and sensory scores were obtained from our clinical evaluation scoring system (Vinik et al 2005b) modified from the Rochester Diabetic Neuropathy Study (Dyck et al 1992). In this system a score of I or 0 is given for the presence or absence of neuropathy symptom respectively. Motor, sensory and autonomic nervous systems are evaluated and given scores on a scale of 0 to 4,0 being no impairment and 4 being severe impairment. Antioxidant treatment instituted at point A, IV Ig infusion instituted at point B and Point $C$ is when gluten free diet was started. 
A gluten-free diet is the main modality of treatment for CD. Other therapies include IVIg and infliximab (Chin and Latov 2005). Although there are reports that gluten-free diet has improved survival and quality of life, facilitated the normal growth and development in infants, prevented osteopenia and decreased the risk of T-cell lymphoma in adults, its effect on the nervous system has been variable (Leffler et al 2003). There are reports where ataxia and PN persisted or even progressed with gluten free diet (Leffler et al 2003; Chin and Latov 2005).

Our patient demonstrates a dichotomy between the responses of $\mathrm{PN}$, autonomic neuropathy and cerebellar ataxia to a gluten-free diet. Nonetheless the frequency of coexistence of $\mathrm{CD}$ in diabetes and the responsiveness of $\mathrm{PN}$ to therapy would encourage a search for the presence of CD in patients with diabetes and neuropathy. Strikingly, the PN improved with measures to reduce autoimmunity supporting the notion that there may be an autoimmune mechanism for damage to peripheral nerves that can be resolved. Unfortunately, this does not appear to be the case for the cerebellar component for reasons that are not apparent at this point in time. The occurrence of cerebellar ataxia emphasizes the need to consider $\mathrm{CD}$ and requires further investigation into possible pathogenetic mechanisms which appear to be distinct from that of the PN.

\section{Disclosure}

The authors report no conflicts of interest in this work.

\section{References}

Aktay AN, Lee PC, Kumar V, et al. 2001. The prevalence and clinical characteristics of celiac disease in juvenile diabetes in Wisconsin. J Pediatr Gastroenterol Nutr, 33:462-5.

Battisti C, Dotti MT, Formichi P, et al. 1996. Disappearance of skin lipofuscin storage and marked clinical improvement in adult onset coeliac disease and severe vitamin E deficiency after chronic vitamin E megatherapy. J Submicrosc Cytol Pathol, 28:339-44.

Bushara KO. 2005. Neurologic presentation of celiac disease. Gastroenterology, 128:S92-S97.

Bushara KO, Goebel SU, Shill H, et al. 2001. Gluten sensitivity in sporadic and hereditary cerebellar ataxia. Ann Neurol, 49:540-3.

Chin RL, Latov N. 2005. Peripheral neuropathy and celiac disease. Curr Treat Options Neurol, 7:43-8.

Chin RL, Sander HW, Brannagan TH, et al. 2003. Celiac neuropathy. Neurology, 60:1581-5.
Dyck PJ, Karnes JL, O’Brien PC, et al. 1992. The Rochester Diabetic Neuropathy Study: reassessment of tests and criteria for diagnosis and staged severity. Neurology, 42:1164-70.

Dyck PJ, Kratz KM, Karnes JL, et al. 1993. The prevalence by staged severity of various types of diabetic neuropathy, retinopathy, and nephropathy in a population-based cohort: the Rochester Diabetic Neuropathy Study. Neurology, 43:817-24.

Granberg V, Ejskjaer N, Peakman M, et al. 2005. Autoantibodies to autonomic nerves associated with cardiac and peripheral autonomic neuropathy. Diabetes Care, 28:1959-64.

Hadjivassiliou M, Gibson A, Davies-Jones GA, et al. 1996. Does cryptic gluten sensitivity play a part in neurological illness? Lancet, 347:369-71

Hadjivassiliou M, Grunewald RA, Chattopadhyay AK, et al. 1998. Clinical, radiological, neurophysiological, and neuropathological characteristics of gluten ataxia. Lancet, 352:1582-5.

Hagen EM, Gjerde IO, Vedeler C, et al. 2000. [Neurological diseases associated with celiac disease]. Tidsskr Nor Laegeforen, 120:439-42.

Honnorat J, Saiz A, Giometto B, et al. 2001. Cerebellar ataxia with antiglutamic acid decarboxylase antibodies: study of 14 patients. Arch Neurol, 58:225-30.

Iwasaki H, Sato R, Shichiri M, et al. 2001. A patient with type 1 diabetes mellitus and cerebellar ataxia associated with high titer of circulating anti-glutamic acid decarboxylase antibodies. Endocr J, 48:261-8.

Koller H, Kieseier BC, Jander S, et al. 2005. Chronic inflammatory demyelinating polyneuropathy. $N$ Engl J Med, 352:1343-56.

Lang B, Dale RC, Vincent A. 2003. New autoantibody mediated disorders of the central nervous system. Curr Opin Neurol, 16:351-7.

Leffler D, Saha S, Farrell RJ. 2003. Celiac disease. Am J Manag Care, 9:825-31.

Luostarinen L, Pirttila T, Collin P. 1999. Coeliac disease presenting with neurological disorders. Eur Neurol, 42:132-5.

Pereira AC, Edwards MJ, Buttery PC, et al. 2004. Choreic syndrome and coeliac disease: a hitherto unrecognized association. Mov Disord, 19:478-82.

Pittenger GL, Liu D, Vinik AI. 1997. The apoptotic death of neuroblastoma cells caused by serum from patients with insulin-dependent diabetes and neuropathy may be Fas-mediated. J Neuroimmunol, 76:153-60.

Sanchez JC, Cabrera-Rode E, Sorell L, et al. 2007. Celiac disease associated antibodies in persons with latent autoimmune diabetes of adult and type 2 diabetes. Autoimmunity, 40:103-7.

Sharma KR, Cross J, Farronay O, et al. 2002. Demyelinating neuropathy in diabetes mellitus. Arch Neurol, 59:758-65.

Vinik A, Ullal J, Parson HK, et al. 2006. Diabetic neuropathies: clinical manifestations and current treatment options. Nat Clin Pract Endocrinol Metab, 2:269-81.

Vinik AI, Anandacoomaraswamy D, Ullal J. 2005a. Antibodies to neuronal structures: innocent bystanders or neurotoxins? Diabetes Care, 28:2067-72.

Vinik AI, Erbas T. 2001. Recognizing and treating diabetic autonomic neuropathy. Cleve Clin J Med, 68:928-44.

Vinik AI, Suwanwalaikorn S, Stansberry KB, et al. 1995. Quantitative measurement of cutaneous perception in diabetic neuropathy. Muscle Nerve, 18:574-84.

Vinik EJ, Hayes RP, Oglesby A, et al. 2005b. The development and validation of the Norfolk QOL-DN, a new measure of patients' perception of the effects of diabetes and diabetic neuropathy. Diabetes Technol Ther, 7:497-508 